\title{
Identifying Long-Run Risks: A Bayesian Mixed-Frequency Approach
}

\author{
Frank Schorfheide* \\ Dongho Song \\ University of Pennsylvania \\ University of Pennsylvania \\ CEPR and NBER
}

\author{
Amir Yaron \\ University of Pennsylvania
}

NBER

February 12, 2013

${ }^{*}$ Correspondence: Department of Economics, 3718 Locust Walk, University of Pennsylvania, Philadelphia, PA 19104-6297. Email: schorf@ssc.upenn.edu (Frank Schorfheide) and donghos@sas.upenn.edu (Dongho Song). The Wharton School, University of Pennsylvania, Philadelphia, PA 19104-6367. Email: yaron@wharton.upenn.edu (Amir Yaron). 


\begin{abstract}
We develop a nonlinear state-space model to capture the joint dynamics of consumption, dividend growth, and asset returns. Building on Bansal and Yaron (2004), the core of our model consists of an endowment economy that is, in part, driven by a common predictable component for consumption and dividend growth. The measurement equations of our state-space model are set up to allow the use of mixed-frequency data, i.e., annual consumption data from 1929 to 1959, monthly consumption data after 1959, and monthly asset return data throughout. Our Bayesian estimation provides strong evidence for a small predictable component in consumption growth (even if asset return data are omitted from the estimation); our measurement error specification implies that consumption is measured much more precisely at annual than monthly frequency; and the estimated model is able to capture key asset pricing facts of the data.
\end{abstract}




\section{Introduction}

Financial economists seek to understand the sources underlying risk and return in the economy. In the context of equilibrium models this endeavor hinges on the joint dynamics for cashflows, which in an endowment economy correspond to consumption and dividends. There are many equilibrium models that appeal to low frequency components in these cashflows as well as important time variation in the fundamentals (e.g., models of long-run risk (LRR) as in Bansal and Yaron (2004), and models of rare disasters as in Barro (2009)). Identifying both of these components is challenging. To measure the small persistent component in, say, consumption and dividend growth one would want the longest span of data. On the other hand, to estimate the time variation in second moments of cashflows one would ideally like to use high frequency data. The empirical analysis is constrained by the availability of consumption data. For the U.S., the longest span of available data for consumption growth is at the annual frequency starting at 1929. The highest frequency consumption data is available at the monthly frequency from 1959. To exploit all the available information in mixed-frequency data, this paper develops a Bayesian state-space model which prominently features stochastic volatility and time-aggregates consumption whenever it is only observed at a low frequency.

Our state-space model is designed to capture the joint dynamics of consumption, dividend growth, and asset returns. Building on the work of Bansal and Yaron (2004), the core of our model consists of an endowment economy that is, in part, driven by a common predictable component for consumption and dividend growth. The economy delivers a stochastic discount factor that is used to price equities and a risk-free asset. Our model distinguishes itself from the existing LRR literature in several important dimensions. First, our statespace representation contains measurement equations which time-aggregate consumption to the observed frequency yet allow us to maintain the likelihood representation (see Bansal, Kiku, and Yaron (2012b) for a GMM approach using time aggregation). Our measurement error specification accounts for different types of measurement errors at monthly and annual frequency while respecting the constraint that monthly growth rates have to be consistent with annual growth rates. Second, we generalize the volatility dynamics of Bansal and Yaron (2004)'s model specification by allowing for three separate volatility processes - one capturing long-run consumption innovations, one capturing short-run consumption innovations, and a separate process for dividend dynamics. We do so since our estimation 
procedure, which focuses on many joint distribution of consumption, dividends, and asset prices, requires separate stochastic volatility processes to fit the data.

The estimation of the state-space model generates several important empirical findings. First, we find strong evidence for a small predictable component in consumption growth. This evidence consists of two parts. We begin by estimating the state-space model on cashflow growth data only. Our carefully specified measurement error model for cashflow data allows us to measure this component which otherwise is difficult to detect. We then proceed by adding asset return data to the estimation and, in line with the existing LRR literature, find even stronger evidence for this predictable component. The Bayesian approach allows us to characterize the uncertainty about the persistence of the conditional mean growth process. We find that in spite of using a prior with a mean of 0.9 and a standard deviation of 0.5 our estimation yields a posterior distribution that is tightly centered around 0.99. Second, our estimated measurement errors for consumption growth are consistent with the common view (see Wilcox (1992)) that consumption growth is measured more precisely at annual than at monthly frequency.

Third, all three stochastic volatility processes display significant time variation yet behave distinctly differently over time. It is important to note that, as emphasized by the LRR literature, the volatility processes have to be very persistent in order to have significant quantitative affects on asset prices. The volatility processes partly capture heteroskedasticity of innovations and in part they break some of the tight links that the model imposes on the conditional mean dynamics of asset prices and cashflows. More specifically, an important feature of our estimation is that the likelihood focus on conditional correlations between risk-free rate and consumption - a dimension often not directly targeted in the literature. We show that because consumption growth and its volatility determines the riskfree rate dynamics, one requires another independent volatility process to account for the weak correlation between consumption growth and the risk-free rate. Fourth, it is worth noting that the median posterior estimate for risk aversion is $7-8$ while it is $1.5-2$ for the intertemporal elasticity of substitution (IES). These estimates are consistent with the parameters highlighted in the LRR literature, see Bansal and Yaron (2004), Bansal, Kiku, and Yaron (2012a), and Bansal, Kiku, and Yaron (2012b). Fifth, at the estimated preference parameters and those characterizing the consumption and dividend dynamics, the model is able to successfully generate many key asset pricing moments. In particular, as in the data, 
the posterior median for the equity premium genereated by the model is $7.5 \%$.

Our paper also contains a number of technical innovations. First, in the specification of our state-space model we follow the stochastic volatility literature and assume that volatilities evolve according to exponential Gaussian processes which guarantee nonnegativity. While the cashflows in our state-space model evolve exogenously, the law of motion of the financial variables is determined endogenously from the economic structure. In order to express the financial variables as functions of the cashflows and volatilities, we have to solve the LRR model. We do so, by approximating the exponential Gaussian volatility processes by linear Gaussian processes such that the standard analytical solution techniques that have been widely used in the LRR literature can be applied. However, the approximation of the exponential volatility process is only used to derive the coefficients in the law of motion of the asset prices. Second, we use a Markov chain Monte Carlo (MCMC) algorithm to generate parameter draws from the posterior distribution. This algorithm requires us to evaluate the likelihood function of our state-space model with a nonlinear filter. Due to the high-dimensional state space that arises from the mixed-frequency setting, this nonlinear filtering is a seemingly daunting task. We show how to exploit the partially linear structure of the state-space model to derive a very efficient sequential Monte Carlo (particle) filter. 


\section{Identifying Long-Run Risks: A Bayesian Mixed-Frequency Approach}

Frank Schorfheide, Dongho Song, and Amir Yaron

University of Pennsylvania

February 12, 2013 


\section{Motivation}

- Financial economists seek to understand the sources of underlying risk and return in the economy

- This endeavor hinges on the joint dynamics for cashflows

- There are many (endowment economy) equilibrium models, e.g., (Bansal and Yaron (2004), Barro (2009)), that appeal to

(1) low frequency variation in the cashflows

(2) important time variation in second moments

- Identifying both of these components is challenging because

(1) one would want to use the longest span of data

(2) but with high frequency data to estimate the time variation in second moments of cashflows

(3) high frequency consumption data are contaminated by measurement errors (Wilcox (1992)) 


\section{Motivation}

- The empirical analysis is constrained by the availability of consumption data

(1) the longest span of available data - annual frequency from 1929

(2) the highest frequency is available at the monthly frequency from 1959

- Building on the work of Bansal and Yaron (2004), we develop a Bayesian state-space model which

(1) prominently features stochastic volatility

(2) carefully specifies measurement error model for cashflows

(3) exploits all the available information in mixed-frequency data

to identify long-run consumption growth and time-varying uncertainty 


\section{Preview of Results - We find...}

(1) strong evidence for a small predictable component in consumption growth, even without asset returns data;

(2) our estimated measurement error specification implies that consumption is measured much more precisely at annual than at monthly frequency;

(3) the estimated model is able to capture many key asset pricing moments of the data;

(4) volatility processes display significant time-variation, need multiple volatility processes to fit data. 
(1) The Long-Run Risks (LRR) model

- Household Preferences and Cashflow Dynamics

- Solution

(2) Taking the LRR model to the Data

- Measurement Error Model for Monthly Consumption Series

- Justification of Measurement Error Model

- Measurement Error Assumptions and Release Schedule

(3) Bayesian Estimation

- State-Space Representation

- Particle Filter MCMC Algorithm

- Empirical Results: Without/With Asset Returns 


\section{LRR Model: Household Preferences}

- Agents maximize life-time utility, which is defined recursively:

$$
V_{t}=\left[(1-\delta) C_{t}^{\frac{1-\gamma}{\theta}}+\delta\left(\mathbb{E}_{t}\left[V_{t+1}^{1-\gamma}\right]\right)^{\frac{1}{\theta}}\right]^{\frac{\theta}{1-\gamma}}
$$

- $\gamma$ is risk aversion; $\theta=\frac{1-\gamma}{1-1 / \psi}$ and $\psi$ is intertemporal elasticity of substitution.

- Budget constraint:

$$
W_{t+1}=\left(W_{t}-C_{t}\right) R_{c, t+1}
$$

where $W_{t}$ is the wealth of the agent, $R_{c, t}$ is the return on all invested wealth. 


\section{LRR Model: Cashflow Dynamics}

- Define consumption growth $g_{t+1}=\ln C_{t+1} / C_{t}$.

- Introduce dividend growth $g_{d, t+1}$.

- Exogenous cashflow processes:

$$
\begin{aligned}
g_{t+1}= & \mu+x_{t}+\sigma_{t} \eta_{t+1} \\
g_{d, t+1}= & \mu_{d}+\phi x_{t}+\pi \sigma_{t} \eta_{t+1}+\sigma_{d, t} u_{t+1} \\
x_{t+1}= & \rho x_{t}+\sigma_{e, t} e_{t+1} \\
& \eta_{t+1}, u_{t+1}, e_{t+1}, w_{h, t+1}, w_{h_{e}, t+1}, w_{h_{d}, t+1} \sim N(0,1) \\
h_{t+1}= & \rho_{h} h_{t}+\sigma_{h} w_{h, t+1}, \quad \sigma_{t}=\bar{\sigma} \exp \left(h_{t}\right) \\
h_{e, t+1}= & \rho_{h_{e}} h_{e, t}+\sigma_{h_{e}} w_{h_{e}, t+1}, \quad \sigma_{e, t}=\varphi_{e} \overline{\exp }\left(h_{e, t}\right) \\
h_{d, t+1}= & \rho_{h_{d}} h_{d, t}+\sigma_{h_{d}} w_{h_{d}, t+1}, \quad \sigma_{d, t}=\varphi_{d} \bar{\sigma} \exp \left(h_{d, t}\right)
\end{aligned}
$$

- $x_{t}$ : a common predictable component. 


\section{LRR Model: Solution}

- The Euler equation for the economy is

$$
\mathbb{E}_{t}\left[\exp \left(\theta \log \delta-\frac{\theta}{\psi} g_{t+1}+(\theta-1) r_{c, t+1}+r_{i, t+1}\right)\right]=1, \quad i \in\{c, m\}
$$

- Campbell and Shiller (1988) approximation: assume

$$
\begin{aligned}
r_{c, t+1} & =\kappa_{0}+\kappa_{1} z_{t+1}-z_{t}+g_{t+1} \\
r_{m, t+1} & =\kappa_{0, m}+\kappa_{1, m} z_{m, t+1}-z_{m, t}+g_{d, t+1}
\end{aligned}
$$

where $z_{t}$ and $z_{m, t}$ are state variables and returns are Gaussian.

- In addition, we approximate the exponential Gaussian vol-processes by linear Gaussian processes

$$
\begin{aligned}
\sigma_{t}^{2} & =\bar{\sigma}^{2} \exp \left(2 h_{t+1}\right), \quad h_{t+1}=\rho_{h} h_{t}+\sigma_{h} w_{h, t+1} \\
\tilde{\sigma}_{t+1}^{2} & =\bar{\sigma}^{2}\left(1-\rho_{h}\right)+\rho_{h} \tilde{\sigma}_{t}^{2}+\left(2 \bar{\sigma}^{2} \sigma_{h}\right) w_{h, t+1} .
\end{aligned}
$$

- Given $\kappa$, law of motion of $z$ and $z_{m}$ can be derived analytically; $\kappa$ 's are constants of log-linearizations which depend on the endogenous mean of $z$ and $z_{m}$. 
- LLR model delivers law of motion for cashflows and asset returns.

- We use measurement equations to link LRR model variables to observables.

- Challenges:

- Consumption data: annual prior to 1959 and monthly post 1959 .

- Monthly consumption data are subject to measurement errors

- Ignoring the measurement errors in monthly consumption makes it impossible to detect the $x_{t}$ process without asset returns.

- For now we will focus on measurement error model for consumption... 


\section{Measurement Error Model for Monthly Consumption Series}

- To economize on notation, suppose that consumption data is released at monthly and/or quarterly (instead of annual) frequency.

- Express the monthly time index $t$ as

$$
t=3(q-1)+m, \quad q=1,2,3,4,5, \ldots, \quad m \in\{1,2,3\}
$$

- Measurement equations for $q=1,2,3,4,5, \ldots$ :

$$
\begin{aligned}
g_{3(q-1)+3}^{o}= & g_{3(q-1)+3}+\epsilon_{3(q-1)+3}-\epsilon_{3(q-1)+2} \\
g_{3(q-1)+2}^{o}= & g_{3(q-1)+2}+\epsilon_{3(q-1)+2}-\epsilon_{3(q-1)+1} \\
g_{3(q-1)+1}^{o}= & g_{3(q-1)+1}+\epsilon_{3(q-1)+1}-\epsilon_{3(q-2)+3} \\
& +\frac{1}{3} \sum_{m=1}^{3}\left(\epsilon_{3(q-1)+m}-\epsilon_{3(q-2)+m}\right)+\epsilon_{3 q}^{Q}-\epsilon_{3(q-1)}^{Q}
\end{aligned}
$$

- Here, $\epsilon_{t}$ 's are monthly measurement errors and $\epsilon_{3 q}^{Q}$ 's are quarterly measurement errors. 


\section{Measurement Error Model for Monthly Consumption Series}

- Average quarterly growth rates can be defined as

$$
\begin{aligned}
g_{3 q}^{o, Q}= & \frac{1}{3} g_{3(q-1)+3}^{o}+\frac{2}{3} g_{3(q-1)+2}^{o}+\frac{3}{3} g_{3(q-1)+1}^{o} \\
& +\frac{2}{3} g_{3(q-2)+3}^{o}+\frac{1}{3} g_{3(q-2)+2}^{o} \\
= & \frac{1}{3} g_{3(q-1)+3}+\frac{2}{3} g_{3(q-1)+2}+\frac{3}{3} g_{3(q-1)+1} \\
& +\frac{2}{3} g_{3(q-2)+3}+\frac{1}{3} g_{3(q-2)+2} \\
& +\epsilon_{3 q}^{Q}-\epsilon_{3(q-1)}^{Q}
\end{aligned}
$$

- Monthly measurement errors average out! 


\section{Conceptual Justification of Measurement Error Model}

Data Construction

\begin{tabular}{cccccccccc}
\hline Quarter & Month & $\begin{array}{c}\text { Quarterly } \\
\text { Release }\end{array}$ & \multicolumn{2}{l}{ Indicator } & Interpolated & $\begin{array}{c}\text { Adjusted } \\
\text { Monthly Release }\end{array}$ \\
\hline 1 & - & $C_{1}^{Q}$ & $(600)$ & $Z_{1}^{Q}$ & $(75)$ & & & & \\
2 & 1 & & & $Z_{2}^{1}$ & $(30)$ & $\tilde{C}_{2}^{1}=C_{1}^{Q} \frac{Z_{2}^{1}}{Z_{1}^{Q}}$ & $(240)$ & $C_{2}^{1}=\tilde{C}_{2}^{1} \frac{C_{2}^{Q}}{\sum_{m=1}^{3} \tilde{C}_{2}^{m}}$ & $(220)$ \\
& 2 & & & $Z_{2}^{2}$ & $(25)$ & $\tilde{C}_{2}^{2}=C_{1}^{Q} \frac{Z_{2}^{2}}{Z_{1}^{Q}}$ & $(200)$ & $C_{2}^{2}=\tilde{C}_{2}^{2} \frac{C_{2}^{Q}}{\sum_{m=1}^{3} \tilde{C}_{2}^{m}}$ & $(183.3)$ \\
& 3 & & & $Z_{2}^{3}$ & $(35)$ & $\tilde{C}_{2}^{3}=C_{1}^{Q} \frac{Z_{2}^{3}}{Z_{1}^{Q}}$ & $(280)$ & $C_{2}^{3}=\tilde{C}_{2}^{3} \frac{C_{2}^{Q}}{\sum_{m=1}^{3} \tilde{C}_{2}^{m}}$ & $(256.7)$ \\
& & & & & & & & & \\
2 & - & $C_{2}^{Q}$ & $(660)$ & $Z_{2}^{Q}$ & $(90)$ & $\tilde{C}_{2}^{Q}=\sum_{m=1}^{3} \tilde{C}_{2}^{m}$ & $(720)$ & & \\
\hline
\end{tabular}

- Write $C_{q}^{m}$ instead of $c_{3(q-1)+m} ; C_{q}^{Q}$ is quarterly consumption in $q$.

- We omit o superscript for observed values. 
- Observed consumption variables are denoted by $C^{\circ}$.

- True consumption is denoted by $C$.

- Measurement errors are denoted by $\epsilon_{q}^{Q}, \epsilon_{q}^{m}$.

- Measurement errors are of multiplicative forms

$$
\begin{aligned}
C_{q}^{Q, o} & =C_{q}^{Q} \exp \left(\epsilon_{q}^{Q}\right) \\
C_{q}^{m, o} & =C_{q}^{Q} \frac{Z_{q}^{m}}{Z_{q}^{1}+Z_{q}^{2}+Z_{q}^{3}} \\
Z_{q}^{m} & =\alpha C_{q}^{m} \exp \left(\epsilon_{q}^{m}\right)
\end{aligned}
$$

- Log-linear approximations for monthly growth rates, e.g., $m=2$

$$
\begin{aligned}
g_{3(q-1)+2}^{o} & =\ln \left(C_{q}^{2, o}\right)-\ln \left(C_{q}^{1, o}\right)=\ln \left(Z_{q}^{2}\right)-\ln \left(Z_{q}^{1}\right) \\
& =g_{3(q-1)+2}+\epsilon_{q}^{2}-\epsilon_{q}^{1}
\end{aligned}
$$




\section{Empirical Justification for Measurement Error Model}

- Our model implies

$$
g_{t+1}=\rho g_{t}+\sigma_{t} \eta_{t+1}+\sigma_{e, t-1} e_{t}-\rho \sigma_{t-1} \eta_{t}+\epsilon_{t+1}-(1+\rho) \epsilon_{t}+\rho \epsilon_{t-1}
$$

- SIC selects an $\operatorname{ARMA}(1,2)$ for monthly consumption series: $A R_{1}=0.92, M A_{1}=-1.16, M A_{2}=0.31$

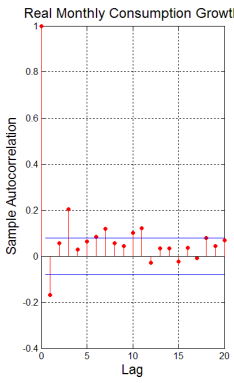

Monthly Data 1959-2011

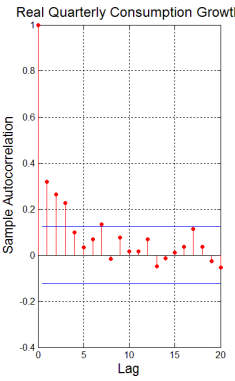

Quarterly Data 1947-2011
Real Annual Consumption Growth

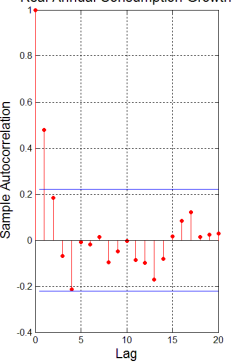

Annual Data

1930-2011 


\section{Bayesian Estimation: Outline}

- State-Space Representation

- Partially Linear State-Space Model

- Particle Filter MCMC Algorithm

- Empirical Results

- Filtered States, Persistence Parameters

- Without Asset Returns, Estimation Sample 1959-2011

- With Asset Returns, Estimation Sample 1929-2011

- Decomposition of Consumption 


\section{State Space Representation: Release Schedule}

- Measurement equations for $q=1,2,3,4,5, \ldots$

$$
\begin{aligned}
g_{3(q-1)+3}^{o}= & g_{3(q-1)+3}+\epsilon_{3(q-1)+3}-\epsilon_{3(q-1)+2} \\
g_{3(q-1)+2}^{o}= & g_{3(q-1)+2}+\epsilon_{3(q-1)+2}-\epsilon_{3(q-1)+1} \\
g_{3(q-1)+1}^{o}= & g_{3(q-1)+1}+\epsilon_{3(q-1)+1}-\epsilon_{3(q-2)+3} \\
& +\frac{1}{3} \sum_{m=1}^{3}\left(\epsilon_{3(q-1)+m}-\epsilon_{3(q-2)+m}\right)+\epsilon_{3 q}^{Q}-\epsilon_{3(q-1)}^{Q}
\end{aligned}
$$

- For a fixed $q$, say $q=2$, three monthly consumption series are released when $m=3$

$$
\underbrace{\left[\begin{array}{l}
N / A \\
N / A \\
N / A
\end{array}\right]}_{m=1} \longrightarrow \underbrace{\left[\begin{array}{l}
N / A \\
N / A \\
N / A
\end{array}\right]}_{(t=4)} \longrightarrow \underbrace{\left[\begin{array}{l}
g_{3(q-1)+3}^{o} \\
g_{3(q-1)+2}^{o} \\
g_{3(q-1)+1}^{o}
\end{array}\right]}_{m=3 \text { or } \quad(t=5)}
$$

Recall: we express the monthly time index $t$ as

$$
t=3(q-1)+m, \quad q=1,2,3,4,5, \ldots, \quad m \in\{1,2,3\}
$$




\section{State-Space Representation: Bayesian Estimation}

Partially Linear Structure:

- Conditional on volatility states, the system becomes linear and Gaussian

- Measurement equation can be written as

$$
Y_{t+1}=M_{t+1}\left(D+Z S_{t+1}+Z^{V} S_{t+1}^{V}+E_{t+1}\right), \quad E_{t+1} \sim N(0, R)
$$

where $M_{t+1}$ is a selection matrix that accounts for the deterministic changes in the data availability

- The state-transition equation is

$$
S_{t+1}=\Phi S_{t}+V_{t+1}
$$

stochastic volatilities are contained in

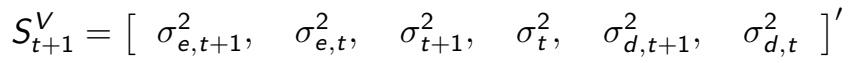




\section{Bayesian Estimation: Particle filter MCMC Algorithm}

1. Draw $\Theta^{j+1} \sim \mathcal{N}\left(\Theta^{j}, \Sigma_{\Theta}\right)$

(a) Transform volatility parameters and obtain pseudo-volatility process, see Appendix B.2

(b) Solve the model, see Appendix C

2. Particle filtering conditional on $\Theta^{j+1}$

(a) Propagate particles $\left\{H_{t+1}^{(i)}\right\}_{i=1}^{N}$ from (A.3) in Appendix B.1

(b) Run Kalman filter using the state-space form in Appendix B.3, conditional on each particle $H_{t+1}^{(i)}$

- Draw $S_{t+1}^{(i)} \sim \mathcal{N}\left(E\left[S_{t+1 \mid t+1}^{(i)}\right], V\left[S_{t+1 \mid t+1}^{(i)}\right]\right)$ where $E\left[S_{t+1 \mid t+1}^{(i)}\right]$ and $V\left[S_{t+1 \mid t+1}^{(i)}\right]$ are the mean and variance of the non-volatility state vector $S_{t+1}$ given $Y_{t+1}$ and $H_{t+1}^{(i)}$

- Evaluate likelihood $p^{(i)}\left(Y_{t+1} \mid S_{t+1}^{(i)}, H_{t+1}^{(i)}\right)$ via the prediction-error decomposition

(c) Update and re-sample the particles, $\left\{S_{t+1}^{(i)}, H_{t+1}^{(i)}, p^{(i)}\left(Y_{t+1} \mid S_{t+1}^{(i)}, H_{t+1}^{(i)}\right)\right\}$, using the probabilities, $\pi_{t}^{(i)}$, see (A.8) in Appendix B.4

(d) Evaluate posterior density

$$
p\left(\Theta^{j+1} \mid Y_{1: T}\right) \propto \sum_{t=0}^{T-1}\left(\sum_{i=1}^{N} \pi_{t}^{(i)} p^{(i)}\left(Y_{t+1} \mid S_{t+1}^{(i)}, H_{t+1}^{(i)}, \Theta^{j+1}\right)\right)+p\left(\Theta^{j+1}\right)
$$

3. Accept $\Theta^{j+1}$ with probability $\min \left\{\frac{\exp \left(p\left(\Theta^{j+1} \mid Y_{1: T}\right)\right)}{\exp \left(p\left(\Theta^{j} \mid Y_{1: T}\right)\right)}, 1\right\}$. Repeat $N_{\text {sim }}$ times. 


\section{Empirical Results: Without Asset Returns, Post 1959}
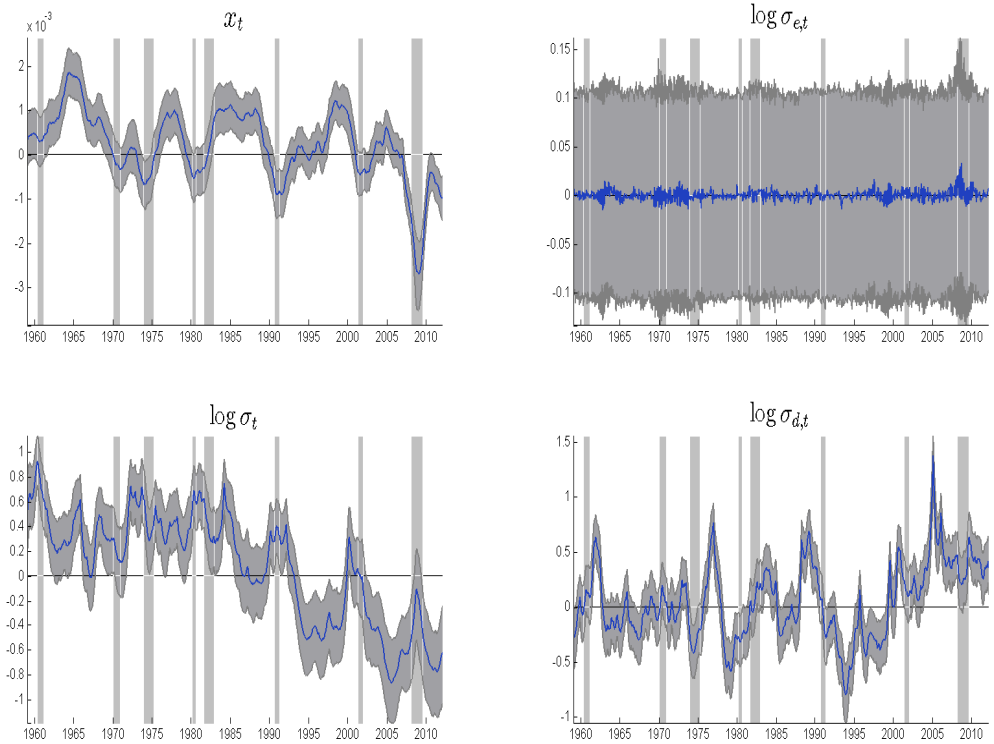
Empirical Results: Without Asset Returns, Post 1959
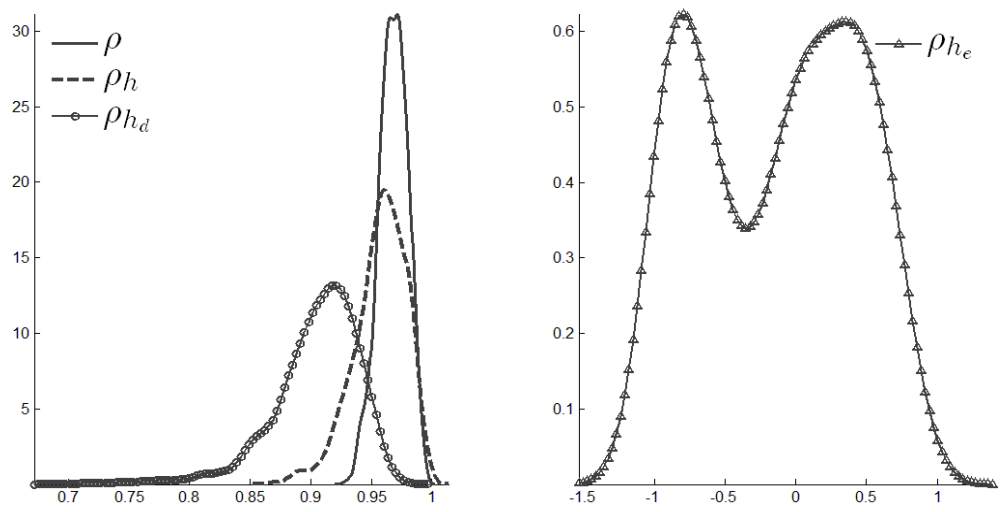


\section{Empirical Results: With Asset Returns, Post 1929}
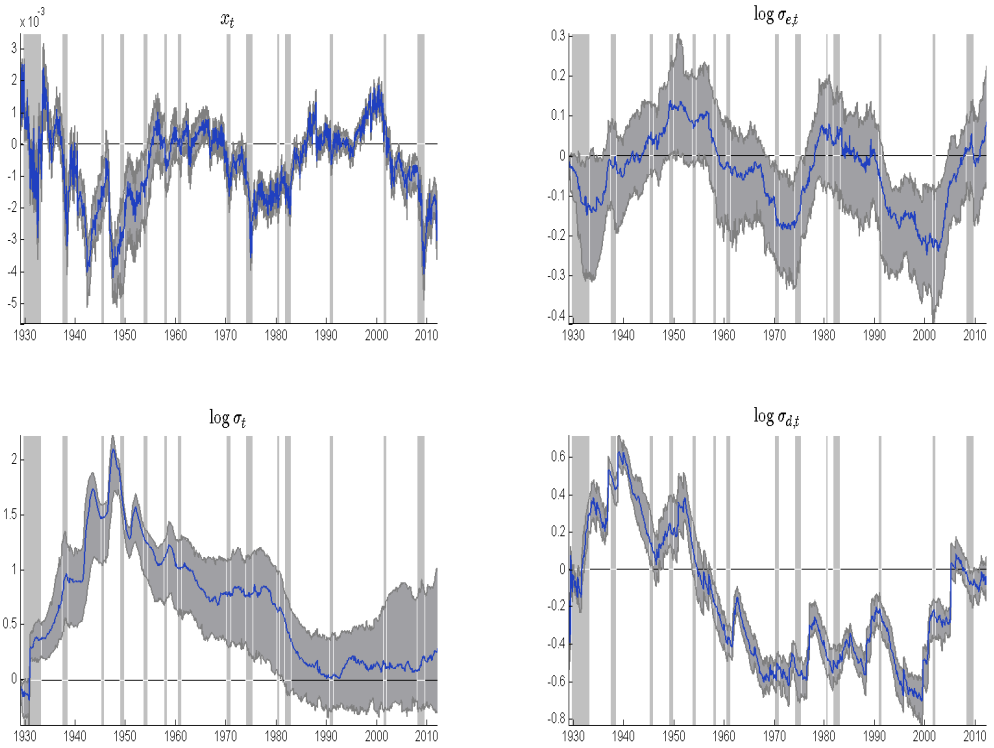


\section{Empirical Results: With Asset Returns, Post 1929}

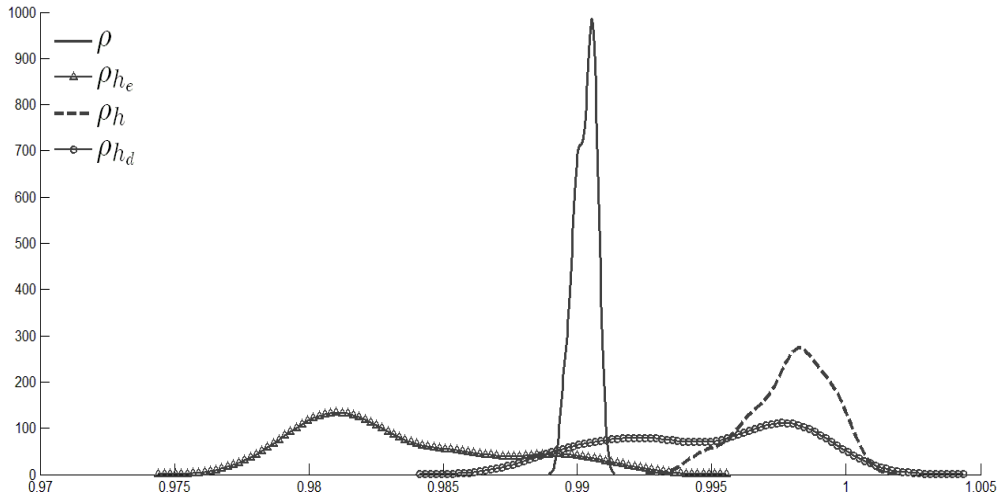




\section{Empirical Results: Posterior Estimates}

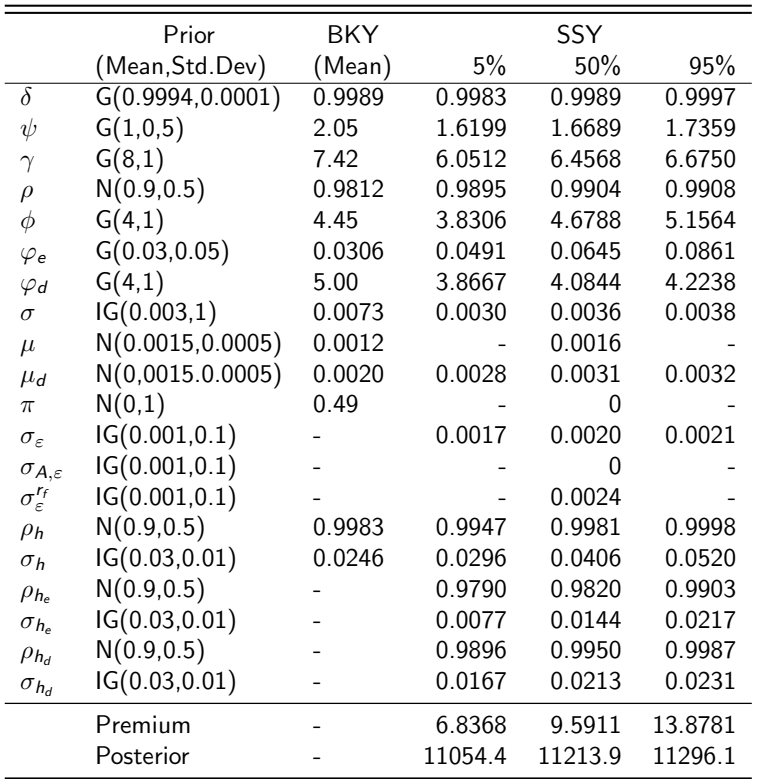




\section{Empirical Results: Decomposition of Consumption}
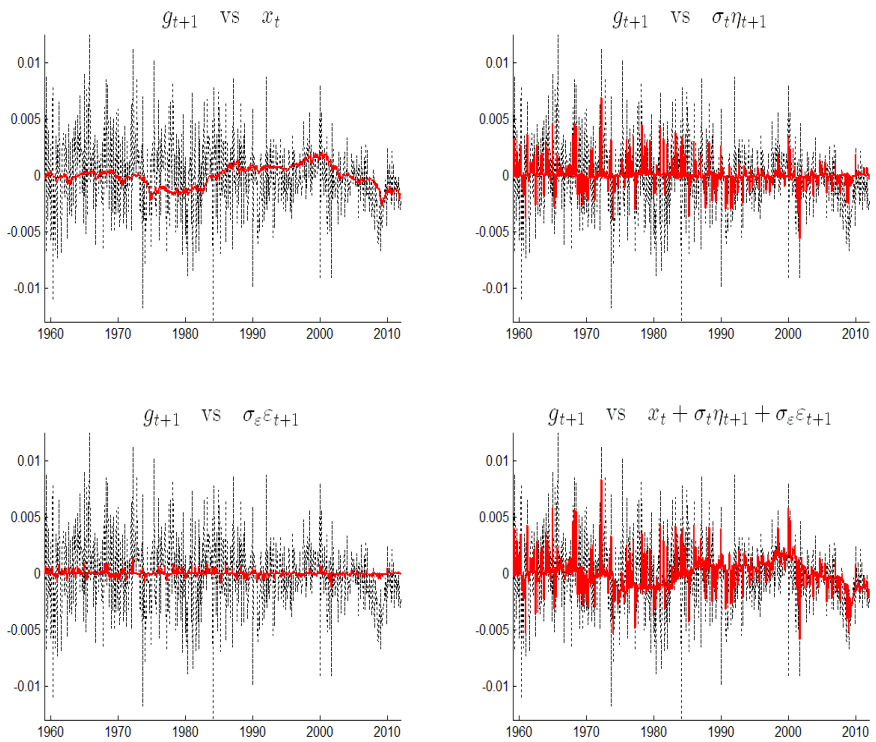


\section{Empirical Results: Decomposition of Consumption}

Table: Variance Decomposition: Measurement Errors

\begin{tabular}{|c|c|c|c|c|c|c|}
\hline \multicolumn{7}{|c|}{ Quarterly Release Scheme: 1959:M1-2011:M12 } \\
\hline & \multicolumn{3}{|c|}{ Without Asset Returns } & \multicolumn{3}{|c|}{ With Asset Returns } \\
\hline & $5 \%$ & $50 \%$ & $95 \%$ & $5 \%$ & $50 \%$ & $95 \%$ \\
\hline$\Delta C_{\text {FirstMonth }}$ & 30.28 & 44.42 & 57.09 & 31.30 & 38.87 & 46.22 \\
\hline$\Delta C_{\text {RemainingMonths }}$ & 36.60 & 47.90 & 58.07 & 25.82 & 32.72 & 41.58 \\
\hline$\Delta C_{Q u a r t e r}$ & 1.19 & 8.44 & 20.35 & 0.81 & 4.51 & 16.75 \\
\hline \multicolumn{7}{|c|}{ Annual Release Scheme: 1959:M1-2011:M12 } \\
\hline & \multicolumn{3}{|c|}{ Without Asset Returns } & \multicolumn{3}{|c|}{ With Asset Returns } \\
\hline & $5 \%$ & $50 \%$ & $95 \%$ & $5 \%$ & $50 \%$ & $95 \%$ \\
\hline$\Delta C_{\text {FirstMonth }}$ & 40.22 & 52.28 & 65.33 & 32.86 & 43.86 & 58.37 \\
\hline$\Delta C_{\text {RemainingMonths }}$ & 37.82 & 48.12 & 57.99 & 29.79 & 37.59 & 44.45 \\
\hline$\Delta C_{\text {Year }}$ & 0.10 & 0.95 & 4.50 & 0.09 & 1.06 & 4.73 \\
\hline
\end{tabular}




\section{Conclusion}

(to be written) 